\title{
GREEN SYNTHESIS OF 2-AMINO-3-CYANO-4H-CHROMENES IN WATER USING NANO SILICA-BONDED 5-N-PROPYL-OCTAHYDRO-PYRIMIDO[1,2-A]AZEPINIUM CHLORIDE AS AN EFFECTIVE AND REUSABLE NANO CATALYST
}

\author{
ROBABEH BAHARFAR*, SAKINEH ASGHARI AND NARGES SHARIATI \\ Department of Chemistry, University of Mazandaran, Babolsar, Iran
}

\begin{abstract}
Nano silica-bonded 5-n-propyl-octahydro-pyrimido[1,2-a]azepinium chloride (NSB-DBU) is reported as an highly efficient and recyclable nano catalyst


environmentally friendly conditions.
\end{abstract}

Keywords: Nano silica-bonded 5-n-propyl-octahydro-pyrimido[1,2-a]azepinium chloride, $4 H$-chromene, green synthesis, aqueous media.

\section{INTRODUCTION}

In recent years, heterogeneous acid and base catalysts have been extensively studied and applied in organic synthesis. ${ }^{1-3}$ They have many advantages compared to homogeneous catalysts, such as their easy recovery and reusability, non-toxicity, non-corrosive, low cost and environmentally benign. ${ }^{4}$ Among them, nano-sized catalysts have attracted more attention than their bulk materials because the size and surface area of nano materials allow the reactions to occur more efficiently than for bulk systems. ${ }^{5,6}$ It is known that surface modification of silica with homogenous catalysts is an excellent method for development of heterogeneous reusable catalysts for organic reactions. ${ }^{7,8}$

The $4 H$-chromene derivatives are the privileged scaffold in the modern medicinal chemistry due to their extensive range of interesting biological activities such as anticancer, antimicrobial, antifungal, antioxidant, antiallergenic, spasmolytic, and anti-anaphylactic. ${ }^{9-17}$ Furthermore, a number of these compounds are utilized as pigments ${ }^{18}$ and photoactive materials. ${ }^{19}$ Several methods have been already reported for the synthesis of these compounds (Table 1). ${ }^{20-32}$ However, these methods are often associated with some drawbacks such as long reaction times, high temperatures, hazardous organic solvents, non-reusable catalysts and complicated work-up. Also, only a few reports existed on the synthesis of these compounds in aqueous media at low temperatures. Therefore, there is still the need to develop better alternatives that can proceed under green reaction conditions.

Table 1. Some examples of reported methods for the synthesis of $4 H$-chromene derivatives.

\begin{tabular}{|c|c|c|c|c|c|c|}
\hline Entry & Catalyst & Solvent & Temp. $/{ }^{\circ} \mathrm{C}$ & Time /min & Yield/\% $\%$ & Ref. \\
\hline 1 & - & Trifluoroethanol & Reflux & 300 & $80-96$ & 20 \\
\hline 2 & $\left(\mathrm{NH}_{4}\right)_{2} \mathrm{HPO}_{4}$ & $\mathrm{H}_{2} \mathrm{O}$ & 25 & $90-300$ & $78-97$ & 21 \\
\hline 3 & $\mathrm{~K}_{3} \mathrm{PO}_{4}$ & $\mathrm{H}_{2} \mathrm{O}: \mathrm{EtOH}(4: 1)$ & 25 & $30-60$ & $87-94$ & 22 \\
\hline 4 & $\mathrm{MgO}$ & $\mathrm{H}_{2} \mathrm{O}: \mathrm{EtOH}(4: 1)$ & Reflux & $22-35$ & $85-96$ & 26 \\
\hline 5 & $\mathrm{RE}(\mathrm{PFO})_{3}$ & $\mathrm{EtOH}$ & 60 & $300-480$ & $81-92$ & 28 \\
\hline 6 & $\mathrm{MSN}^{\mathrm{a}}$ & $\mathrm{EtOH}$ & 60 & $10-45$ & $93-98$ & 29 \\
\hline 7 & - & {$[\mathrm{BmIm}] \mathrm{OH}^{\mathrm{b}}$} & 25 & $5-20$ & $85-98$ & 30 \\
\hline 8 & - & {$[\mathrm{omim}] \mathrm{PF}_{6}^{\mathrm{c}}$} & 25 & $120-480$ & $52-94$ & 31 \\
\hline 9 & $\mathrm{TBAF}^{\mathrm{d}}$ & $\mathrm{H}_{2} \mathrm{O}$ & Reflux & 30 & $73-98$ & 32 \\
\hline
\end{tabular}

\footnotetext{
${ }^{a}$ Mesoporous Silica Nanoparticles.

b 1-Butyl-3-methylimidazolium hexafluorophosphate.

c 1-Methyl-3-octylimidazolium hexafluorophosphate.

d Tetrabutylammonium fluoride.
}

Recently, we prepared silica-bonded nano silica-bonded 5-n-propyloctahydro-pyrimido[1,2-a] azepinium chloride (NSB-DBU) (Scheme 1) as a solid base nano catalyst for the synthesis of novel benzothiazole substituted 4-thiazolidinones. ${ }^{33}$ In continuation of our previous work to develop new and eco-friendly synthetic methodologies in organic synthesis, ${ }^{33,36}$ we report herein the application of nano silica-bonded 5-n-propyl-octahydro-pyrimido[1,2-a] azepinium chloride (NSB-DBU) as a heterogeneous and easy recoverable catalyst in the green synthesis of $4 H$-chromene derivatives (Scheme 2 ).



Scheme 1. Preparation of NSB-DBU 


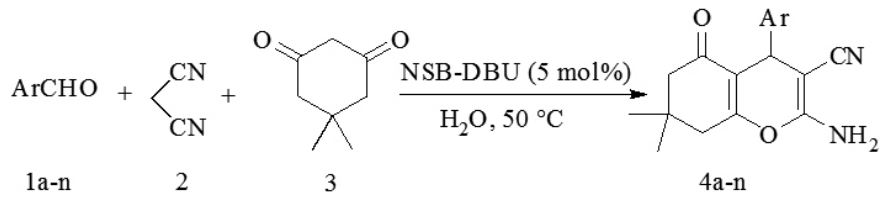

Scheme 2. Synthesis of $4 H$-chromene derivatives.

\section{RESULTS AND DISCUSSION}

First, NSB-DBU was prepared from the reaction of nano silica-npropyl chloride and DBU as shown in Scheme 1. The catalyst structure was characterized by X-ray diffraction (XRD), Fourier-transfer infrared (FTIR) spectroscopy, field emission scanning electron microscopy (FE-SEM), termogravimetric analysis (TGA) and elemental analysis (CHN). XRD diffractogram of NSB-DBU showed strong broad peak between $22^{\circ}$ and $23^{\circ}$

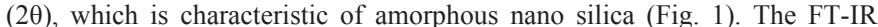
diagram of NSB-DBU ( $\mathrm{sp}^{3} \mathrm{C}-\mathrm{H}$ stretching at $2936 \mathrm{~cm}^{-1}, \mathrm{C}=\mathrm{N}$ stretching at $1647 \mathrm{~cm}^{-1}, \mathrm{CH}_{2}$ bending at $1449 \mathrm{~cm}^{-1}$ and Si-O stretching at $1099 \mathrm{~cm}^{-1}$ as a broad band) was in complete agreement with the proposed structure (Fig. 2). From the multiple SEM images, it can be determined that the average diameter of $\mathrm{SiO}_{2}$ nanoparticles after immobilization of DBU is about $40 \mathrm{~nm}$ (Fig. 3).

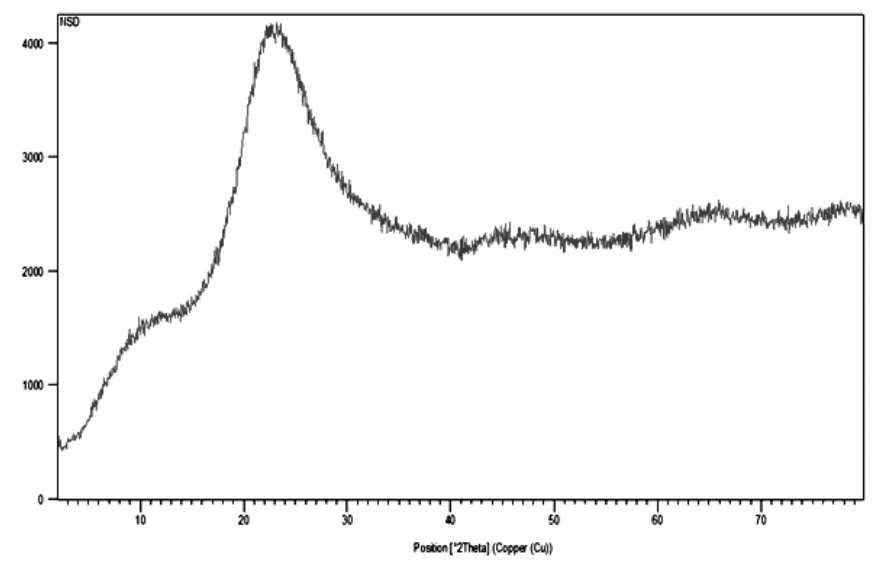

Figure 1. XRD pattern of NSB-DBU

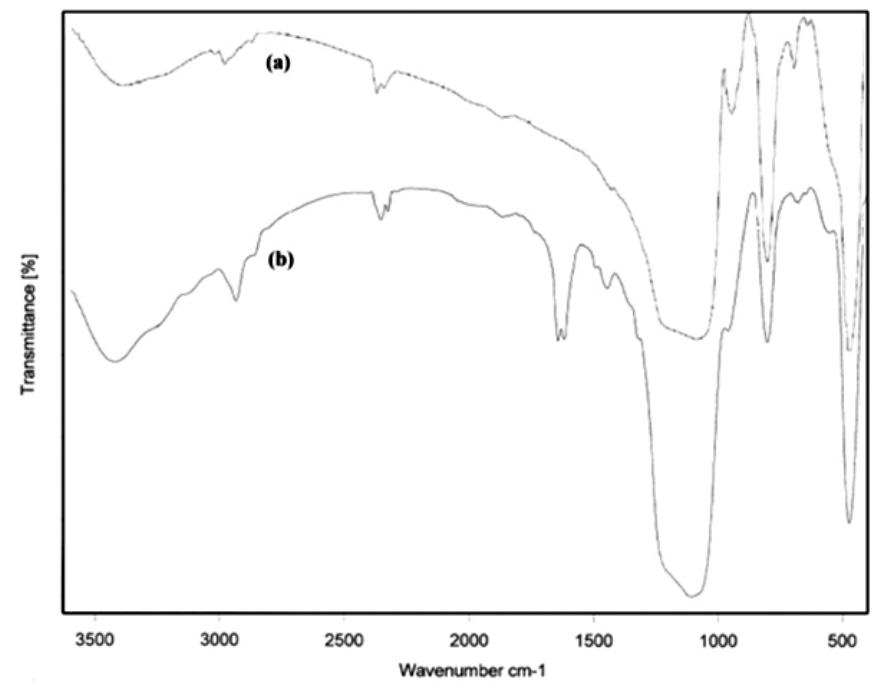
DBU

Figure 2. FT-IR spectra of (a) nano silica-n-propyl chloride and (b) NSB-

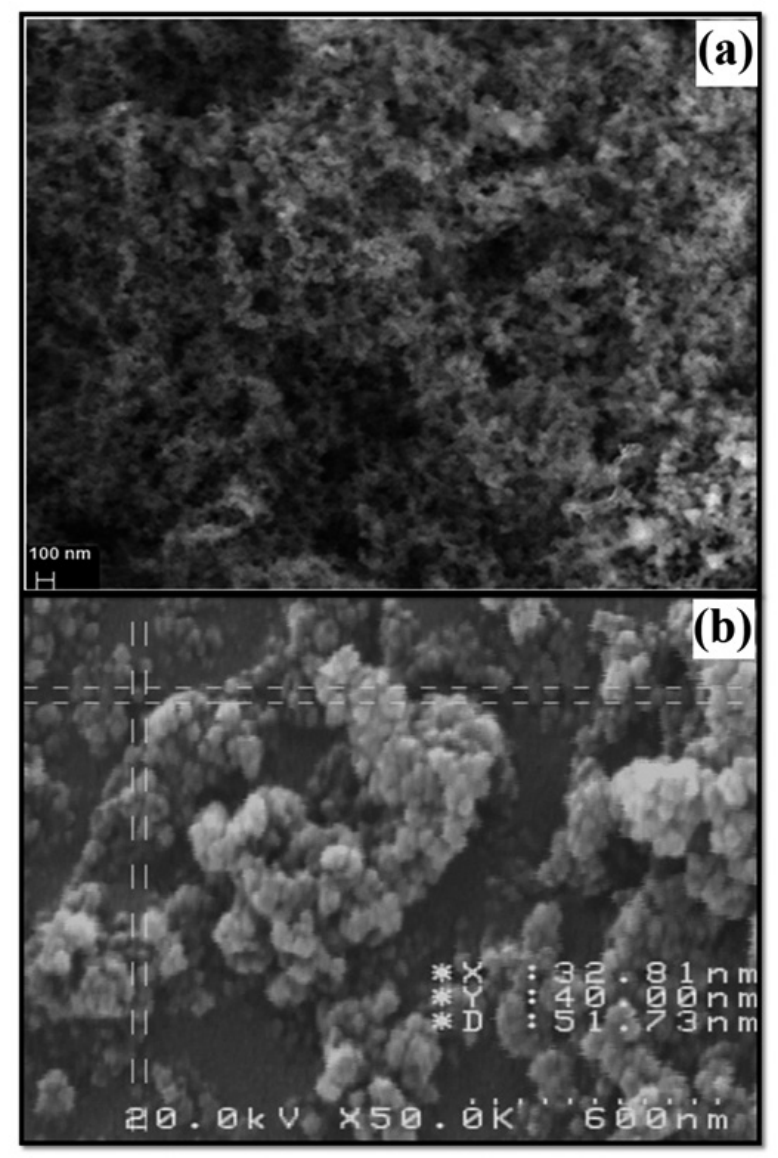

Figure 3. FE-SEM of (a) Nano silica and (b) NSB-DBU

The amount of DBU grafted on nano silica was evaluated by the nitrogen content, $1.15 \mathrm{mmol} / \mathrm{g}$, on the basis of elemental analysis (C: $16.92 \% \mathrm{H}: 3.06 \%$ $\mathrm{N}: 3.22 \%$ ). This amount was significantly higher than the loading level of DBU on silica gel as reported earlier $(0.45 \mathrm{mmol} / \mathrm{g}){ }^{38}$ Thermogravimetric (TG) analysis of NSB-DBU was also studied. The lost weight around $200{ }^{\circ} \mathrm{C}$ can be related to the loss of the organic moiety (DBU) of NSB-DBU (Fig. 4). NSBDBU showed almost 26\% weight loss in this area (about $1.14 \mathrm{mmol}$ of DBU per $1.0 \mathrm{~g}$ of the catalyst) which was in accordance with the results obtained from elemental analysis.

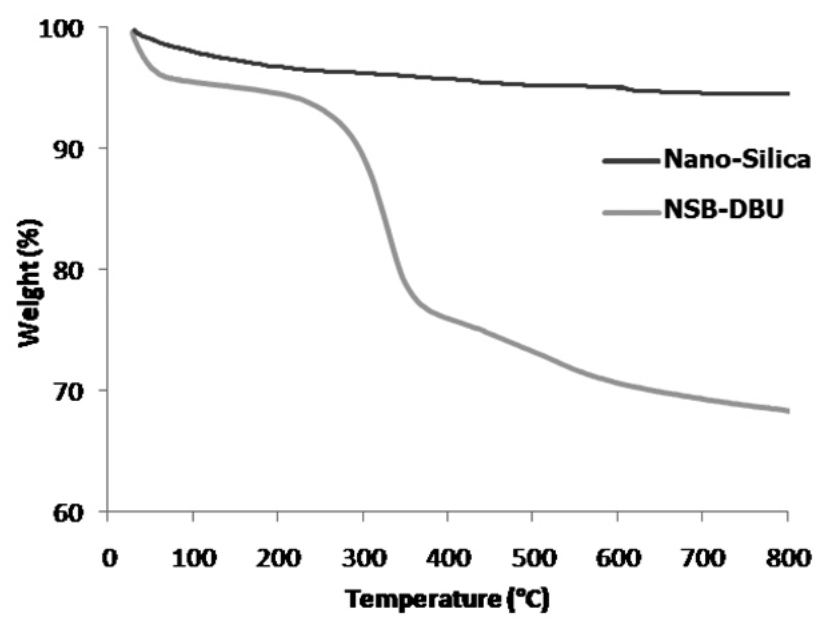

Figure 4. Thermal gravimetric analysis of nano silica and NSB-DBU 
In order to evaluate the efficiency of NSB-DBU as catalyst, threecomponent synthesis of 2-amino-3-cyano-4H-chromenes was studied. For the optimization of the reaction conditions, the reaction of benzaldehyde, dimedone and malononitrile was chosen as a model reaction (Table 2). First, we studied the ability of different catalysts including nano $\mathrm{SiO}_{2}$, nano $\mathrm{MgO}$, nano $\mathrm{TiO}_{2}$, nano $\mathrm{ZnO}$ and $\mathrm{DBU}$ in the synthesis of 2-amino-3-cyano-4H-chromene derivatives. As shown in Table 1, the reaction did not take place without any catalyst (Table 1, entry 1). It was observed that most interesting result was obtained with NSB-DBU as the catalyst. The best results were achieved by carrying out the reaction of benzaldehyde, dimedone and malononitrile (with a 1:1:1 molar ratio) in the presence of $5 \mathrm{~mol} \%$ of NSB-DBU in water at 50 ${ }^{\circ} \mathrm{C}$. Subsequently, a series of differently substituted tetrahydrobenzo[b]pyran derivatives was prepared successfully under aqueous conditions (Table 3 ). The results clearly indicate that reactions can tolerate a wide range of differently substituted aldehydes. The reactions were complete within 20-40 min and excellent yields of products were obtained by this method. The aromatic aldehydes with electron withdrawing groups reacted faster with slightly improved yields than their electron donating counter parts (Table 3, entry 4-6). However, in case of aliphatic aldehydes, reactions were not complete under the above conditions and desired products were obtained in low yields.

Table 2. Optimization of reaction conditions ${ }^{\mathrm{a}}$

\begin{tabular}{|c|c|c|c|c|c|}
\hline Entry & Catalyst (mol \%) & Solvent & Temperature $/{ }^{\circ} \mathrm{C}$ & Time/min & Yields $/ \%^{\mathrm{b}}$ \\
\hline 1 & - & $\mathrm{H}_{2} \mathrm{O}$ & 50 & 180 & Trace \\
\hline 2 & $\mathrm{SiO}_{2}-\mathrm{NPs}^{\mathrm{c}}(10)$ & $\mathrm{H}_{2} \mathrm{O}$ & 50 & 160 & 20 \\
\hline 3 & $\mathrm{MgO}-\mathrm{NPs}(10)$ & $\mathrm{H}_{2} \mathrm{O}$ & 50 & 160 & 35 \\
\hline 4 & $\mathrm{TiO}_{2}-\mathrm{NPs}(10)$ & $\mathrm{H}_{2} \mathrm{O}$ & 50 & 180 & 30 \\
\hline 5 & $\mathrm{ZnO}-\mathrm{NPs}(10)$ & $\mathrm{H}_{2} \mathrm{O}$ & 50 & 180 & 30 \\
\hline 6 & DBU (10) & $\mathrm{H}_{2} \mathrm{O}$ & 50 & 75 & 45 \\
\hline 7 & NSB-DBU (20) & $\mathrm{H}_{2} \mathrm{O}$ & 50 & 30 & 93 \\
\hline 8 & NSB-DBU (10) & $\mathrm{H}_{2} \mathrm{O}$ & 50 & 15 & 93 \\
\hline 9 & NSB-DBU (5) & $\mathrm{H}_{2} \mathrm{O}$ & 50 & 20 & 93 \\
\hline 10 & NSB-DBU (5) & $\mathrm{THF}^{2}$ & 50 & 120 & 48 \\
\hline 11 & NSB-DBU (5) & $\mathrm{CHCl}_{3}$ & 50 & 70 & 25 \\
\hline 12 & NSB-DBU (5) & $\mathrm{CH}_{2} \mathrm{Cl}$ & 50 & 55 & 21 \\
\hline 13 & NSB-DBU (5) & $\mathrm{EtOH}_{2}$ & 50 & 35 & 94 \\
\hline 14 & NSB-DBU (5) & $\mathrm{CH}_{3} \mathrm{CN}$ & 50 & 40 & 32 \\
\hline 15 & NSB-DBU (5) & $\mathrm{H}_{2} \mathrm{O}$ & r.t. & 30 & 78 \\
\hline 16 & NSB-DBU (5) & $\mathrm{H}_{2} \mathrm{O}$ & reflux & 15 & 93 \\
\hline
\end{tabular}

${ }^{a}$ Reaction and conditions: benzaldehyde $(1 \mathrm{mmol})$, dimedone $(1 \mathrm{mmol})$, malononitrile $(1 \mathrm{mmol})$, different conditions, stirring.

${ }^{\mathrm{b}}$ Isolated yields.

${ }^{\mathrm{c}}$ NPs: Nano particles.

Table 3. Synthesis of 2-amino-4H-benzopyran derivatives in aqueous media

\begin{tabular}{|c|c|c|c|c|c|c|}
\hline \multirow{2}{*}{ Entry } & \multirow{2}{*}{ Aldehyde } & \multirow{2}{*}{ Product } & \multirow{2}{*}{ Time/min } & \multirow{2}{*}{ Yield/ $\%{ }^{\mathrm{b}}$} & \multicolumn{2}{|c|}{ Melting point $/{ }^{\circ} \mathrm{C}$} \\
\hline & & & & & Found & Reported \\
\hline 1 & $\mathrm{PhCHO}$ & $4 a$ & 20 & 93 & $233-235$ & $234-235^{32}$ \\
\hline 2 & 2-Cl-PhCHO & $4 \mathrm{~b}$ & 25 & 91 & $212-213$ & $214-215^{39}$ \\
\hline 3 & 4-Cl-PhCHO & $4 c$ & 20 & 92 & $216-218$ & $215-217^{40}$ \\
\hline 4 & $2-\mathrm{NO}_{2}-\mathrm{PhCHO}$ & $4 d$ & 30 & 91 & $221-222$ & $224-226^{41}$ \\
\hline 5 & $3-\mathrm{NO}_{2}-\mathrm{PhCHO}$ & $4 \mathrm{e}$ & 20 & 95 & $211-212$ & $212-214^{41}$ \\
\hline 6 & $4-\mathrm{NO}_{2}-\mathrm{PhCHO}$ & $4 \mathrm{f}$ & 25 & 93 & $176-178$ & $179-180^{42}$ \\
\hline 7 & 2-Br-PhCHO & $4 \mathrm{~g}$ & 35 & 88 & $151-152$ & $150-152^{43}$ \\
\hline 8 & 4-Br-PhCHO & $4 \mathrm{~h}$ & 30 & 94 & $201-203$ & $203-205^{43}$ \\
\hline 9 & 4-OMe-PhCHO & $4 \mathrm{i}$ & 30 & 90 & $199-200$ & $201-202^{32}$ \\
\hline 10 & 4-Me-PhCHO & $4 j$ & 40 & 86 & $229-231$ & $223-225^{42}$ \\
\hline 11 & 4-CN-PhCHO & $4 \mathrm{k}$ & 30 & 89 & $208-210$ & $208-210^{42}$ \\
\hline 12 & $4-\mathrm{Me}_{2} \mathrm{~N}-\mathrm{PhCHO}$ & 41 & 35 & 87 & $213-215$ & $210-212^{44}$ \\
\hline 13 & 4-F-PhCHO & $4 \mathrm{~m}$ & 35 & 88 & 206-209 & $210-211^{38}$ \\
\hline 14 & 4-OH-PhCHO & $4 n$ & 30 & 90 & $204-205$ & $206-208^{43}$ \\
\hline 15 & $\mathrm{CH}_{3}\left(\mathrm{CH}_{2}\right)_{2} \mathrm{CHO}$ & 40 & 480 & 12 & - & - \\
\hline 16 & $\mathrm{CH}_{3} \mathrm{CH}_{2} \mathrm{CHO}$ & $4 p$ & 480 & 5 & - & - \\
\hline
\end{tabular}

a condition: Benzaldehyde derivative $(1 \mathrm{mmol})$, malononitrile $(1 \mathrm{mmol})$, dimedone $(1 \mathrm{mmol}), \mathrm{NSB}-\mathrm{DBU}(5 \mathrm{~mol} \%, 0.05 \mathrm{~g}), \mathrm{H}_{2} \mathrm{O}(5 \mathrm{~mL}), 50{ }^{\circ} \mathrm{C}, \mathrm{stirring}$.

${ }^{\mathrm{b}}$ Isolated yield. 




Figure 5. Reusability of the NSB-DBU in the synthesis of $\mathbf{4 a}$

A proposed mechanism for the synthesis of $4 H$-chromenes is outlined in Scheme 3. Initially, NSB-DBU catalyzed the readily in situ formation of Knoevenagel product 5. Afterward, enolate form of carbonyl compound 3 reacts with adduct $\mathbf{5}$ to produce intermediate $\mathbf{6}$, which converts to product $\mathbf{4}$, after proton transfer, cyclization and tautomerization.



Scheme 3. Proposed mechanism for the preparation of $4 H$-chromenes.

\section{EXPERIMENTAL}

All chemicals and reagents were purchased from Fluka and Merck and used without further purification. Melting points were measured on an Electrothermal 9100 apparatus. NMR spectra were recorded with a Bruker DRX-400 AVANCE instrument (400.1 MHz for ${ }^{1} \mathrm{H}, 100.6 \mathrm{MHz}$ for ${ }^{13} \mathrm{C}$ ) with $\mathrm{CDCl}_{3}$ or DMSO- $\mathrm{d}_{6}$ as solvent. IR spectra were recorded on an FT-IR Bruker vector 22 spectrometer. Thermogravimetric analysis (TGA) was recorded on a Stanton Redcraft STA-780 (London, UK). The XRD was performed using PHILIPS Xpert pro diffractometer with $\mathrm{Cu} K \alpha$ radiation $\left(\lambda 1.54 \mathrm{~A}^{\circ}\right)$. FE-SEM images were obtained on a Hitachi S-1460 field emission scanning electron microscope using an ACC voltage of $15 \mathrm{kV}$.

3.1. Preparation of nano silica-bonded 5-n-propyl-octahydropyrimido[1,2-a]azepinium chloride (NSB-DBU)

NSB-DBU was prepared according to our previously reported procedure. ${ }^{33}$ First, nano silica-n-propyl chloride was prepared as reported earlier. ${ }^{37}$ Then, a mixture of nano silica-n-propyl chloride $(1.0 \mathrm{~g})$ and 1,8-Diazabicyclo[5.4.0] undec-7-ene (DBU) $(0.76 \mathrm{~g}, 5.0 \mathrm{mmol})$ in cyclohexane $(30 \mathrm{~mL})$ was added to a $50 \mathrm{~mL}$ round bottomed flask connected to a reflux condenser. The mixture was stirred under reflux conditions for $36 \mathrm{~h}$. The resulting mixture was then filtered, extracted with toluene in a Soxhlet extractor for $24 \mathrm{~h}$ and dried at 60 ${ }^{\circ} \mathrm{C}$ in vacuo to give NSB-DBU as a white powder $(1.3 \mathrm{~g})$.

3.2. Synthesis of 2-amino-5-oxo-5,6,7,8-tetrahydro- $4 \mathrm{H}$-chromenes (4an)

A mixture of aldehyde derivatives $(1 \mathrm{mmol})$, malononitrile $(1 \mathrm{mmol})$, dimedone ( $1 \mathrm{mmol}$ ) and NSB-DBU $(5 \mathrm{~mol} \%, 0.05 \mathrm{~g})$ in $5 \mathrm{~mL} \mathrm{H} \mathrm{O}$ was stirred at $50{ }^{\circ} \mathrm{C}$ until completion of reaction (TLC-control). Then, the solid was dispersed in hot ethanol, and the catalyst was removed by filtration. After cooling, the crude product was precipitated and then purified by recrystallization from ethanol. The recovered catalyst was dried and reused in further reactions.

3.3. Selected spectral data

2-Amino-7,7-dimethyl-5-oxo-4-phenyl-5,6,7,8-tetrahydro-4H-chromene3-carbonitrile $(4 a)$. White powder; m.p.: $233-235^{\circ} \mathrm{C}$; yield $(93 \%)$; IR $(\mathrm{KBr})$ $v_{\max }: 1681(\mathrm{C}=\mathrm{O}), 2200(\mathrm{CN}), 2961\left(\mathrm{H}-\mathrm{Csp}^{3}\right), 3214(\mathrm{~N}-\mathrm{H}), 3323(\mathrm{~N}-\mathrm{H}) \mathrm{cm}^{-1}$; ${ }^{1} \mathrm{H}$ NMR $(400 \mathrm{MHz}, \mathrm{DMSO}-\mathrm{d}) \delta: 0.96\left(\mathrm{~s}, 3 \mathrm{H}, \mathrm{CH}_{3}\right), 1.04\left(\mathrm{~s}, 3 \mathrm{H}, \mathrm{CH}_{3}\right), 2.10$ and $2.26\left(2 \mathrm{~d}, J=16.0 \mathrm{~Hz}, 2 \mathrm{H}, \mathrm{CH}_{2}\right), 2.50-2.52\left(\mathrm{~m}, 2 \mathrm{H}, \mathrm{CH}_{2}\right), 4.17(\mathrm{~s}, 1 \mathrm{H}, \mathrm{CH})$, $7.01\left(\mathrm{~s}, 2 \mathrm{H}, \mathrm{NH}_{\mathrm{r}}\right), 7.13-7.17(\mathrm{~m}, 3 \mathrm{H}, 3 \mathrm{CH}), 7.29(\mathrm{t}, J=7.2 \mathrm{~Hz}, 2 \mathrm{H}, 2 \mathrm{CH})$; ${ }^{13} \mathrm{C}$ NMR (100 MHz, DMSO-d $\left.{ }_{6}\right) 27.3,28.9,32.3,36.0,39.6,50.4,58.7,113.2$, $120.2,127.0,127.6,128.8,145.2,158.9,163.0,196.1$.

2-Amino-4-(2-chlorophenyl)-7,7-dimethyl-5-oxo-5,6,7,8-tetrahydro-4Hchromene-3-carbonitrile (4b). White powder; m.p.: $212-213{ }^{\circ} \mathrm{C}$; yield $(91 \%)$; IR $(\mathrm{KBr}) v: 1673(\mathrm{C}=\mathrm{O}), 2188(\mathrm{CN}), 3253(\mathrm{~N}-\mathrm{H}), 3372(\mathrm{~N}-\mathrm{H}) \mathrm{cm}^{-1} ;{ }^{1} \mathrm{H}$ NMR $\left(400 \mathrm{MHz}, \mathrm{DMSO}-\mathrm{d}_{6}\right) \delta: 0.92\left(\mathrm{~s}, 3 \mathrm{H}, \mathrm{CH}_{3}\right), 1.03\left(\mathrm{~s}, 3 \mathrm{H}, \mathrm{CH}_{3}\right), 2.09$ and 2.22 $\left(2 \mathrm{~d}, J=16.0 \mathrm{~Hz}, 2 \mathrm{H}, \mathrm{CH}_{2}\right), 2.52\left(\mathrm{~s}, 2 \mathrm{H}, \mathrm{CH}_{2}\right), 4.32(\mathrm{~s}, 1 \mathrm{H}, \mathrm{CH}), 7.03(\mathrm{~s}, 2 \mathrm{H}$, $\left.\mathrm{NH}_{2}\right), 7.12\left(\mathrm{t}, J=8.7 \mathrm{~Hz}, 2 \mathrm{H}, 2 \mathrm{CH}_{\mathrm{A}}\right), 7.14-7.22\left(\mathrm{~m}, 2 \mathrm{H}, 2 \mathrm{CH}_{\mathrm{r}}\right) ;{ }^{13} \mathrm{C} \mathrm{NMR}$ $\left(100 \mathrm{MHz}, \mathrm{DMSO}-\mathrm{d}_{6}\right) \delta: 27.4,28.6,32.3,35.1,50.2,58.6,113.5,115.4,115.8$, $119.7,129.5,129.6,140.6,141.3,158.8,160.2,162.4,196.3$.

\section{CONCLUSIONS}

In conclusion, highly efficient and environmentally green methodology for the synthesis of $4 \mathrm{H}$-chromene derivatives via the three-component reaction of aromatic aldehydes, malononitrile and dimedone has been developed in excellent yields (87-95\%) within short reaction times (20-40 min). This protocol has several advantages including mild conditions, excellent yield, environmentally benign and an easy work-up procedure without the need for column chromatographic purification. The catalyst can be recovered and reused over several reaction cycles without considerable loss of reactivity.

\section{ACKNOWLEDGMENTS}

This research was supported by the Research Council of the University of Mazandaran, Iran.

\section{REFERENCES}

1. T. Yokoi, Y. Kubota, T. Tatsumi, Appl. Catal. A. 14, 421, (2012).

2. Y. Tang, G. Chen, Y. Lu, Res. Chem. Intermediate 38, 937, (2012).

3. G. Szöllösi, M. Bartók, J. Mol. Struct. 482, 13, (1999)

4. R.S. Varma, Green Chem. 1, 43, (1999).

5. A. Chrobok, S. Baj, W. Pudlo, A. Jarzebski, Appl. Catal., A. 366, 22, (2009).

6. Q. Zhang, H. Su, J. Luo, Y. Wei, Green Chem. 14, 201, (2012). 
7. S. Safaei, I. Mohammadpoor-Baltork, A.R. Khosropour, M. Moghadam, S. Tangestaninejad, V. Mirkhani, Catal. Sci. Technol. 3, 2717, (2013).

8. B.S.G. Taki, V. Mirkhani, I. Mohammadpoor-Baltork, M. Moghadam, S. Tangestaninejad, M. Rostami, A.R. Khosropour, J. Inorg. Organomet. Polym. Mater. 23, 758, (2013).

9. W.P.Smith, L.S. Sollis, D.P. Howes, C.P. Cherry, D.I. Starkey, N.K. Cobley, J. Med. Chem. 41, 787, (1998).

10. A.G. Martinez, L.J. Marco, Bioorg. Med. Chem. Lett. 7, 3165, (1997).

11. G. Bianchi, A. Tava, Agric. Boil. Chem. 51, 2001, (1987).

12. S.J. Mohr, M.A. Chirigos, F.S. Fuhrman, J.W. Pryor, Cancer research 35 , 3750, (1975)

13. F.M. Abdelrazeka, P. Metza, E.K. Farragb, Arch. Pharm. 337, 482, (2004).

14. L. Bonsignore, G. Loy, D. Secci, A. Calignano, Eur. J. Med. Chem. 28, 517, (1993).

15. N.R. Kamdar, D.D. Haveliwala, P.T. Mistry, S.K. Patel, Eur. J. Med. Chem. 45, 5056, (2010).

16. W. Kemnitzer, S. Kasibhatla, S. Jiang, H. Zhang, J. Zhao, S. Jia, L. Xu, C. Crogan- Grundy, R. Denis, N. Barriault, L. Vaillancourt, S. Charron, J. Dodd, G. Attardo, D. Labrecque, S. Lamothe, H. Gourdeau, B. Tseng, J. Drewea, S.X. Caia, Bioorg. Med. Chem. Lett. 15, 4745, (2005).

17. L.L. Andreani, E. Lapi, Boll. Chim. Farm. 99, 583, (1960).

18. G.P. Ellis, in The chemistry of heterocyclic compounds chromenes, chromanes and chromones, A. Weissberger and E.C. Taylor, Eds. John Wiley, New York, NY, 1977; pp. 11-139.

19. D. Armesto, W.M. Horspool, N. Martin, A. Ramos, C. Seoane, J. Org. Chem. 54, 3069, (1989).

20. S. Khaksar, A. Rouhollahpour, S.M. Talesh, J. Fluorine Chem. 141, 11, (2012).

21. S. Balalaie, M. Bararjanian, M. Sheikh-Ahmadi, S. Hekmat, P. Salehi, Syn. Comm. 37, 1097, (2007).

22. D.M. Pore, K.A. Undale, B.B. Dongare, U.V. Desai, Catal. Lett. 132, 104, (2009).
23. T.S. Jin, A. Wang, X. Wang, J.S. Zhang, T.S. Li, Synlett 2004, 871, (2004).

24. S. Banerjee, A. Horn, H. Khatri, G. Sereda, Tetrahedron Lett. 52, 1878, (2011).

25. A. Saini, S. Kumar, J.S. Sandhu, Synlett 2006, 1928, (2006).

26. M. Seifi, H. Sheibani, Catal. Lett. 126, 275, (2008).

27. X.Z. Lian, Y. Huang, Y.Q. Li, W.J. Zheng, Monatsh. Chem. 139, 129, (2008).

28. L.M. Wang, J.H. Shao, H. Tian, Y.H. Wang, B. Liu, J. Fluorine Chem. 127, 97, (2006).

29. Y. Sarrafi, E. Mehrasbi, A. Vahid, M. Tajbakhsh, Chin. J. Catal. 33, 1486, (2012).

30. B.C. Ranu, S. Banerjee, S. Roy, Indian J. Chem. B. 47, 1108, (2008).

31. Z.Q. Jiang, S.J. Ji, J. Lu, J.M. Yang, Chin. J. Chem. 23, 1085, (2005).

32. S. Gao, C.H. Tsai, C. Tseng, C.F. Yao, Tetrahedron 64, 9143, (2008).

33. R. Baharfar, N. Shariati, Aust. J. Chem., 67,1646, (2014).

34. R. Baharfar, N. Shariati, C. R. Chimie 17, 413, (2014).

35. N. Shariati, R. Baharfar, J. Chin. Chem. Soc. 61, 337, (2014)

36. R. Baharfar, S. Peiman, M. Mohseni, Lett. Org. Chem. 11, 393, (2014).

37. X. Zhang, Y. Zhang, Y. Yang, Q. Wei, X. Zhang, React. Kinet. Catal. Lett. $94,385,(2008)$.

38. A. Hasaninejad, N. Golzar, M. Beyrati, A. Zare, M.M. Doroodmand, J. Mol. Cat. A. 372, 137, (2013).

39. D. Fang, H.B. Zhang, Z.L. Liu, J. Heterocycl. Chem. 47, 63, (2010).

40. M. Kazemzad, A.A. Yuzbashi, S. Balalaie, M. Bararjanian, Synth. React. Inorg. Metal-Org. nano-Met. Chem. 41, 1182, (2011).

41. T.S. Jin, L.B. Liu, Y. Zhao, T.S. Li, Synth. Commun. 35, 1859, (2005)

42. S. Balalaie, M. Bararjanian, A.M. Amani, B. Movassagh, Synlett 2006, 263, (2006).

43. S.J. Tu, Y. Gao, C. Guo, D. Shi, Z. Lu, Synth. Commun. 32, 2137, (2002).

44. T.A. Khan, M. Lal, S. Ali, M.M. Khan, Tetrahedron Lett. 52, 5327, (2011). 